Ficco, C. \& Luna, J. (2021). Divulgación de información no financiera sobre capital intelectual en el reporte integrado: un estudio de caso en el mercado de capitales argentino. Contaduría Universidad de Antioquia, 79, 39-62.

Doi: https://doi.org/10.17533/udea.rc.n79a02

\title{
Divulgación de información no financiera sobre capital intelectual en el reporte integrado: un estudio de caso en el mercado de capitales argentino
}

Cecilia Ficco

Universidad Nacional de Río Cuarto cficco@fce.unrc.edu.ar Orcid: 0000-0003-0474-9978

Jonathan Luna Valenzuela

Universidad Nacional de Río Cuarto jvalenzuela@fce.unrc.edu.ar Orcid: 0000-0002-3041-3565 
Divulgación de información no financiera sobre capital intelectual en el reporte integrado: un estudio de caso en el mercado de capitales argentino

Resumen: El objetivo fundamental de este trabajo es analizar la divulgación de información no financiera sobre capital intelectual (CI) en el reporte integrado (RI). Para dar cumplimiento al objetivo planteado se realiza un estudio descriptivo, adoptando el enfoque de estudio de caso, que permite analizar, en las prácticas de divulgación de información, aquella que refiere al CI y que se canaliza a través del RI. El estudio empírico se sustenta en el análisis de datos textuales y se realiza sobre una empresa perteneciente al sector financiero, pionera en la adopción del RI en el mercado de capitales argentino. Las evidencias obtenidas indican que la empresa analizada divulga información sobre CI y sus dimensiones, humana, estructural y relacional, en el RI; adoptando la estructura del marco del IIRC. Estos hallazgos apoyan la idea de que el CI está resurgiendo como parte del RI.

Palabras Clave: Capital intelectual - Divulgación voluntaria - Información no financiera - Reporte integrado - Estudio de caso.

Disclosure of non-financial intellectual capital information in integrated reporting: a case study in the Argentine capital market

Abstract: The main aim of this paper is to analyze the disclosure of non-financial information on intellectual capital (IC) in integrated reporting (IR). To achieve the stated goal, a descriptive study is conducted using the case study approach, which allows to analyze, in information disclosure practices, the information that refers to IC and is channeled through IR. The empirical study is based on textual data analysis and is conducted on a financial sector company that has been pioneer in the adoption of $I R$ in the Argentine capital market. The evidence obtained indicates that the company analyzed discloses information on IC and its human, structural and relational dimensions in the IR, adopting the structure of the IIRC framework. These findings support the idea that IC is re-emerging as part of IR.

Keywords: Intellectual capital - Voluntary disclosure - Non-financial information - Integrated reporting - Case study.

Divulgação de informação não financeira sobre capital intelectual no relatório integrado: um estudo de caso no mercado de capitais argentino

Resumo: $O$ objetivo fundamental deste trabalho é analisar a divulgação de informação não financeira sobre capital intelectual (CI) no relatório integrado (RI). Para cumprir o objetivo planteado se realiza um estudo descritivo, adotando o foco de estudo de caso, que permite analisar, nas práticas de divulgação de informação, aquela que refere ao CI e que se canaliza a través do RI. O estudo empírico sustenta-se na análise de dados textuais e realiza-se sobre uma empresa pertencente ao setor financeiro, pioneira na adoção do RI no mercado de capitais argentino. As evidências obtidas indicam que a empresa analisada divulga informação sobre CI e suas dimensões, humana, estrutural e relacional, no RI; adotando a estrutura do marco do IIRC. Estes descobrimentos apoiam a ideia de que o CI está ressurgindo como parte do RI.

Palavras Chave: Capital intelectual; divulgação voluntária; informação não financeira; relatório integrado; estudo de caso.

Divulgation d'Informations non financières sur le capital intellectuel dans les rapports intégrés : une étude de cas sur le marché financier argentin

Résumé: Cet article vise à analyser la divulgation d'informations non financières sur le capital intellectuel (CI) dans le rapport intégré (RI). Afin d'atteindre l'objectif fixé, une étude descriptive est réalisée, en adoptant une approche d'étude de cas, qui nous permet d'analyser, dans les pratiques de divulgation, ce qui se réfère au CI, et qui est canalisé par le RI. L'étude empirique est basée sur l'analyse de données textuelles et est réalisée sur une entreprise appartenant au secteur financier, pionnière dans l'adoption du RI sur le marché financier argentin. Les preuves obtenues indiquent que l'entreprise analysée divulgue des informations sur le CI et ses dimensions humaines, structurelles et relationnelles dans le RI, en adoptant la structure du cadre de l'International Integrated Reporting Council (IIRC). Ces résultats soutiennent l'idée que le CI réapparaît comme une partie du RI.

Mots clés: Capital intellectuel, Divulgation volontaire, Information non financière, Rapport intégré, Étude de cas. 
Cont. udea (julio-septiembre, pp. 39-62. (C) Universidad de Antioquia-2021.

\title{
Divulgación de información no financiera sobre capital intelectual en el reporte integrado: un estudio de caso en el mercado de capitales argentino
}

\author{
Cecilia Ficco y Jonathan Luna Valenzuela \\ https://doi.org/10.17533/udea.rc.n79a02
}

Primera versión recibida en abril de 2021 - Versión aceptada en julio de 2021

\section{Introducción}

En el entorno actual en el que se desenvuelven las organizaciones, los recursos intangibles asumen un papel central en la generación de valor (Ficco, Werbin, Díaz y Prieto, 2021a). Se trata, tal y como señala Cañibano (2012, p. 43), de "los nuevos creadores de valor empresarial y fuente de ventaja competitiva", por lo que constituyen recursos estratégicos para las empresas (Bueno, Salmador y Merino, 2008). Estos elementos habitualmente se engloban bajo la denominación de "capital intelectual" (CI), el cual representa un concepto amplio que abarca los conocimientos y las habilidades de los empleados, las rutinas y los procedimientos organizativos, el capital tecnológico, la capacidad de innovación, las relaciones con los clientes y otros stakeholders, la reputación de la empresa en el mercado, entre otros (Ficco, 2019; Rossi, Festa, Chouaibi, Fait y Papa, 2021).

La contabilidad financiera presenta información limitada con relación a estos elementos, ya que los organismos emisores de normas han adoptado, en general, una postura restrictiva en lo que respecta a la inclusión de los intangibles en los estados financieros, debido a las dificultades que presenta su identificación y medición (García-Meca, 2005; Pastor, Glova, Lipták y Kovác, 2017; Sharma, 2012).

Esta deficiencia informativa hace necesario otro tipo de información, complementaria a los estados financieros, como podrían ser los informes de $\mathrm{CI}$, los cuales servirían para comunicar a terceros la relevancia de los recursos y actividades intangibles de las empresas y su potencialidad para generar valor (Cañibano, 2012). No obstante, aunque se han desarrollado diferentes 
Divulgación de información no financiera sobre capital intelectual en el reporte integrado...

propuestas para la elaboración de este tipo de informes, no existe un marco general que rija el contenido y guíe su preparación. Tampoco se ha generalizado su uso por parte de las empresas a nivel voluntario (Dumay, 2016).

En este contexto, la revelación de información voluntaria sobre recursos intangibles es la práctica que más auge ha cobrado entre las empresas, como alternativa para dar a conocer su CI (Herrera y Macagnan, 2016). Para ello, se emplean diversos formatos y canales (Castilla y Ruiz, 2018). Por un lado, se utilizan las notas a los estados financieros y la memoria, en las cuales se incluye, principalmente, información no financiera, de tipo cualitativo, que complementa a la información referida a los elementos intangibles que tienen reconocimiento como activos (Ficco, García, Sader y Luna, 2021b). Por otro lado, las memorias de sostenibilidad, elaboradas bajo los estándares de Global Reporting Initiative (GRI) y, más recientemente, el reporte integrado (RI), propuesto por el International Integrated Reporting Council (IIRC), constituyen otros medios que permiten a las organizaciones revelar información no financiera que tiene relación estrecha con los elementos intangibles clave de la generación de valor público y organizacional (Ficco y Sader, 2020).

En particular, el RI se vislumbra como una solución prometedora frente a las limitaciones de los informes financieros tradicionales (Busco, Malafronte, Pereira y Starita, 2019; Dey, 2020). Y, en ese marco, también parece erigirse como una alternativa para la comunicación de información sobre $\mathrm{CI}$, a tal punto que algunos autores, como Abhayawansa, Guthrie y Keynes (2019), postulan que el surgimiento del RI se encuentra estrechamente vinculado a la falta de progreso de las iniciativas de informes específicos de $\mathrm{Cl}$, destacando que el $\mathrm{RI}$ ha reivindicado la importancia del $\mathrm{CI}$ de las empresas.

Específicamente, el RI es un informe que tiene como propósito principal explicar "cómo una organización crea valor a lo largo del tiempo" (IIRC, 2013, p. 11). El marco del IIRC identifica seis capitales, a saber: "financiero, industrial, intelectual, humano, social y relacional, y natural" (IIRC, 2013, p. 12), que refieren a "los recursos y las relaciones utilizados y afectados por la organización" (IIRC, 2013, p. 11) en el proceso de generación de valor, involucrando tanto a bienes físicos como a recursos y actividades intangibles (Ficco et al., 2021b). De este modo, el $\mathrm{CI}$, tal como lo concibe la literatura específica, es decir, como concepto holístico que abarca todas las formas de elementos intangibles (Cañibano, Sánchez, García y Chaminade, 2002) de las organizaciones, parece tener una importancia central en el RI.

Sin embargo, como afirman Santis, Bianchi, Incollingo y Bisogno (2019), son pocos los estudios que se han concentrado en la importancia del $\mathrm{CI}$ dentro del $\mathrm{RI}$, indagando acerca de su contribución a la creación de valor, por lo que es necesario aumentar la investigación en esta línea, a efectos de desarrollar nuevos conocimientos sobre el CI (Dumay y Cai, 2014). 
En el ámbito latinoamericano las deficiencias en este campo son aún mayores, ya que ni siquiera se ha generalizado el uso del RI como práctica corporativa de divulgación de información (Macías y Farfán, 2017; Rivera, Zorio y García, 2016). En Argentina los RI (al igual que las memorias de sostenibilidad) son de presentación voluntaria y son escasas las empresas que los elaboran. Específicamente, en el contexto del mercado de capitales argentino, solo tres empresas, pertenecientes al sector financiero, elaboran RI a partir del marco de referencia del IIRC. En particular, el Banco Macro S.A. (BMA) fue la primera empresa en presentar un RI en Argentina en 2014. Las otras dos empresas referidas hacen lo propio desde el año 2018.

De esta manera, en el marco del problema informativo existente en relación con el $\mathrm{Cl}$ de las empresas y teniendo en cuenta que la investigación previa deja una importante laguna sin cubrir, debido a la escasez de estudios sobre la divulgación del $\mathrm{Cl}$ en el informe integrado, el presente trabajo tiene como objetivo fundamental analizar cómo se realiza la divulgación de información no financiera sobre $\mathrm{CI}$ en los $\mathrm{RI}$, evaluando, además, si la revelación del $\mathrm{Cl}$ se ajusta al marco del IIRC o si se encuentra más en línea con la concepción de la literatura específica sobre $\mathrm{CI}$.

Para el cumplimiento al objetivo planteado, se adopta el enfoque del estudio de caso (Ryan, Scapens y Theobald, 2004; Yin, 2009), para lo cual se seleccionó a la empresa pionera en elaboración de RI en Argentina: el BMA. La pertenencia de esta empresa al sector financiero, único sector de actividad de Argentina en el que se registran empresas cotizantes que elaboran RI, brinda un ámbito propicio para el estudio de la revelación del $\mathrm{Cl}$, debido a la importancia que el mismo posee en dicho sector, puesto que se trata de uno de los más intensivos en conocimiento y donde el $\mathrm{Cl}$ asume especial relevancia para la creación de valor (Irsyahma y Nikmah, 2017; Saengchan, 2008; Veltri y Silvestri, 2011). Además, permite aportar al conocimiento de un sector que, pese a su relevante papel en la economía, ha sido menos estudiado en las investigaciones previas (Castilla y Ruiz, 2018; Mention, 2011).

Para desarrollar el estudio se analizan los RI del BMA elaborados para el período 2014-2019. El estudio se sustenta en el análisis de datos textuales, lo cual le confiere originalidad al presente trabajo, ya que no se ha constatado la existencia de otros grupos de investigación que la utilicen dentro de la línea de estudios sobre $\mathrm{CI}$, ni tampoco dentro de la referida al RI, siendo escasos los trabajos que la aplican en otras áreas disciplinares de las Ciencias Económicas (Benites, Mello y Lahsen, 2017).

Así, después de esta introducción, el desarrollo del trabajo se ha estructurado en tres grandes secciones. En la primera, se presenta el marco teórico de la investigación, el que se integra con el marco conceptual del $\mathrm{Cl}$, en la literatura específica y en el RI, y con la revisión de la literatura empírica previa. En 
Divulgación de información no financiera sobre capital intelectual en el reporte integrado...

la segunda, se describe la metodología del estudio empírico realizado y, en la tercera, se presentan los resultados del mismo y su discusión. Finamente, se exponen las conclusiones que se derivan de tales resultados, las limitaciones de la investigación y sus perspectivas futuras.

\section{Marco teórico y revisión de la literatura}

\section{II.1 Marco conceptual del capital intelectual en la literatura específica}

Tal como se indicaba en la introducción, el CI representa una categoría que incluye a las distintas formas de elementos intangibles que pueden tener las empresas. Sin embargo, no existe consenso en torno a su concepto en la literatura específica sobre $\mathrm{CI}$, en la que se han propuesto distintas definiciones, con diferentes alcances en cuanto a los elementos que involucra. Esta situación refleja la complejidad de un concepto que es multifacético (Ficco, 2020) y que aún puede considerarse como emergente y en rápida evolución.

No obstante, a través de la revisión de la literatura, se detectan algunas características que son comunes a la mayoría de los conceptos elaborados, por lo que, tal como señalan Martín, Navas, López y Delgado (2010), pueden considerarse definitorias del $\mathrm{Cl}$. En este sentido, el $\mathrm{Cl}$ se considera como un conjunto de elementos de carácter intangible relacionados entre sí, que comprende no solo recursos sino también actividades de esta naturaleza (Cañibano et al., 2002; Sveiby, 1997), y que permiten a la empresa funcionar, en combinación con los demás recursos inherentes a la misma (Bueno et al., 2008), siendo esenciales para la creación de valor y la construcción y sostenimiento de la ventaja competitiva (Cañibano et al., 2002; Edvinsson y Malone, 1997; Lentjušenkova y Lapina, 2016; Ross, Ross, Dragonetti y Edvinson, 2001; Sardo y Serrasqueiro, 2018).

De este modo, el $\mathrm{Cl}$ incluye elementos de diversa índole, los que, habitualmente, se engloban en tres grandes dimensiones: capital humano $(\mathrm{CH})$, capital estructural (CE) y capital relacional (CR), siendo estas categorías las que definen los grandes componentes del $\mathrm{Cl}$.

El CH refiere al valor intangible que reside en los empleados de la firma (Santis et al., 2019) e incluye los saberes, capacidades, experiencias y habilidades que poseen los mismos (Cañibano et al., 2002), siendo, esencialmente, conocimiento tácito (Bontis, 1998; Nonaka, 1991).

El CE es la infraestructura que incorpora, forma y sostiene al CH (Edvinsson y Malone, 1997). Incluye rutinas organizativas, procedimientos, sistemas y estructuras de la organización que ayudan a optimizar el rendimiento intelectual de los empleados (Bontis, 1998; Santis et al., 2019). El CE permite la conversión del conocimiento individual en colectivo (Bollen, Vergauwen y Schnieders, 2005), por lo que se vincula con la idea de conocimiento explícito (Nonaka, 1991). Es 
importante señalar que involucra también los elementos que aluden a la capacidad de renovación de la empresa y los resultados de la innovación (Ochoa, Prieto y Santidrián, 2010), algunos de cuales "pueden protegerse legalmente y convertirse en derechos de propiedad intelectual o industrial, como los derechos de autor o las patentes" (Cañibano et al., 2002, p. 19).

El CR está constituido por los elementos intangibles que pueden generar valor a partir de las relaciones que la empresa entabla con su ámbito externo (Ginesti, Caldarelli y Zampella, 2018) y refiere a la red de relaciones con diferentes stakeholders (Ross et al., 2001). Representa, así, una dimensión esencialmente externa a la empresa (Sveiby, 1997), que incluye también los nombres de sus productos, las marcas y su reputación o imagen (Ficco, 2020).

\section{II.2 El capital intelectual en el reporte integrado}

El RI es una iniciativa del IIRC que, en 2013, presentó el Marco Internacional del Reporte Integrado (MIRI) para orientar su elaboración. En particular, el RI es un informe que tiene como propósito principal "explicar a los proveedores de capital financiero cómo la organización crea valor a lo largo del tiempo. Por lo tanto, contiene información relevante, tanto financiera como de otro tipo" (IIRC, 2013, p. 8).

Bajo el enfoque del RI se considera que "el valor no es creado por o dentro de una organización por sí solo" (IIRC, 2013, p. 11), sino que la generación de valor es influenciada por el entorno, se produce a través de las relaciones con los grupos de interés y depende de diversos recursos (IIRC, 2013).

Por ello, el RI brinda información sobre el entorno externo y sobre los recursos y las relaciones utilizados y afectados por la organización (denominados como capitales), explicando, además, cómo interactúa con el entorno externo y con los capitales para crear valor en el corto, medio y largo plazo (IIRC, 2013). Es, de esta manera, "una comunicación concisa sobre el modo en que la estrategia de una organización, así como su gobierno, desempeño y perspectivas, en el contexto de su entorno, conducen a la creación de valor en el corto, medio y largo plazo" (IIRC, 2013, p. 35).

Los capitales asumen una importancia central en el enfoque del RI, en la medida en que la creación de valor se concibe como el proceso que "se manifiesta en un aumento, disminución o transformación de los capitales causados por las actividades y las salidas de la organización" (IIRC, 2013, p. 11). Los capitales son, en definitiva:

[...] los inventarios de valor de los que depende el éxito de cualquier empresa, ya que, de una forma u otra, son recursos de su modelo de negocio, y aumentan, disminuyen o se transforman a través de las actividades y la producción de la organización. (IIRC, 2013, p. 35). 
Divulgación de información no financiera sobre capital intelectual en el reporte integrado...

Los capitales, en el MIRI, se agrupan en seis categorías: "Financiero, Industrial, Intelectual, Humano, Social y Relacional, y Natural” (IIRC, 2013, p. 5), las cuales se describen de la forma que se expone en la Figura 1:

Figura 1. Capitales del MIRI.

Capital Financiero
Fondos a los que el ente accede para la elaboración de sus productos o prestaciones de
servicios, incluidos los fondos obtenidos de fuentes externas.

Capital Industrial

Bienes tangibles que están disponibles para la elaboración de los productos o prestaciones de servicios; incluye bienes inmovilizados e infraestructura.

Capital Intelectual
Intangibles basados en el conocimiento de la organización, incluyendo la propiedad
intelectual y el capital organizacional.

Capital Social y Relacional

Instituciones y relaciones dentro y entre las comunidades, los grupos de interés y otras redes y la capacidad de compartir información para mejorar el bienestar individual y colectivo.

Capital Natural
Recursos renovables y no renovables que el ente utiliza para la elaboración de sus
productos o prestaciones de servicios, y los procesos medioambientales que sostienen
la prosperidad de la organización.

Fuente: IIRC (2013).

Se puede apreciar, como lo hacen notar Santis et al. (2019), que, en el MIRI, el Capital Humano y el Capital Social y Relacional se toman como capitales separados del Intelectual, lo que difiere de la categorización usada en la literatura específica del CI. No obstante, es posible efectuar una asociación, a nivel conceptual, entre los componentes de los capitales que se describen en el MIRI y las tres dimensiones en las que habitualmente se engloban los componentes del $\mathrm{CI}(\mathrm{CH}$, CE y CR). Así, el Capital Intelectual del MIRI, que reúne los inventarios de valor asociados a los intangibles basados en el conocimiento de la organización, se relaciona con la dimensión estructural del CI. Del mismo modo, es posible identificar que los Capitales Humano y Social y Relacional se encuentran conceptualmente vinculados a las dimensiones del CI que reciben análogas denominaciones (Badia, Dicuonzo, Petruzzelli y Dell'Atti, 2019; Melloni, 2015; Zambon, 2016).

Por otro lado, Ficco et al. (2021b) destacan que algunas de las categorías de capital, descritas por el MIRI, no guardan estrecha relación con las dimensiones del CI. Los autores lo atribuyen al hecho de que el modelo de creación de valor 
que presenta el MIRI incluye, además, elementos de carácter tangible considerados necesarios para la producción de bienes o la prestación de servicios, como es el caso de los bienes inmovilizados y la infraestructura (Capital Industrial), junto a los recursos financieros (Capital Financiero) y a los recursos naturales renovables y no renovables (Capital Natural), que no estarían involucrados en la concepción clásica del $\mathrm{CI}$, de acuerdo a la cual él mismo engloba elementos de carácter intangible. No obstante, indican que el Capital Natural incluye, además de los recursos naturales, los procesos medioambientales; procesos que sí pueden asociarse a la dimensión estructural del $\mathrm{Cl}$, en tanto resultan representativos del compromiso de la organización con el medio ambiente.

\section{II.3 Revisión de la literatura empírica previa}

La literatura sobre divulgación de información no financiera, y en particular sobre intangibles, se ha incrementado notablemente en los últimos años, abarcando estudios de variada naturaleza. Entre los trabajos que se encuentran más directamente vinculados al objeto de esta investigación se ubican aquellos centrados en describir las prácticas voluntarias de divulgación de $\mathrm{Cl}$ adoptadas por las empresas, los que se han centrado, principalmente, en la cantidad de información que es revelada. En esta línea se incluyen los llevados a cabo por Al-Hajaya, Altarawneh y Altarawneh (2019), Blaise, Carson y Philips (2008), De Silva, Stratford y Clark (2014), Mention (2011), entre otros, la mayoría de los cuales concluye sobre la tendencia creciente a divulgar información sobre intangibles de manera voluntaria por parte de las empresas.

En lo que respecta a los medios o canales de comunicación utilizados en el análisis, los estados financieros constituyen el documento más revisado al analizar la divulgación de CI (Ferchichi y Paturel, 2013; Sihotang y Sanjaya, 2014; Tejedo, 2016), lo que ha dado lugar, tal como señalan Castilla y Ruiz (2018), a una de las principales críticas que ha recibido la literatura existente en esta materia. En este sentido, Dumay y Cai (2014) han enfatizado la necesidad de estudiar la divulgación de información sobre $\mathrm{CI}$ en el contexto del RI, lo que puede resultar especialmente relevante si se tiene en cuenta que existe abundante evidencia sobre la influencia que posee el $\mathrm{Cl}$ en la generación de valor.

Así, una importante línea de estudios, tendiente a demostrar la relevancia valorativa de la información referida al $\mathrm{Cl}$, ha examinado la asociación entre dicha información y alguna medida del valor de las empresas. Estos trabajos han utilizado tanto información financiera sobre $\mathrm{Cl}$, principalmente referida a los activos intangibles reconocidos contablemente, como información no financiera (Ficco y Sader, 2020). Estas investigaciones aportan evidencias sólidas en lo atinente a la influencia positiva de los activos intangibles identificables y de los elementos integrantes del $\mathrm{CH}$ y del $\mathrm{CE}$, no reconocidos contablemente, en el valor de mercado de las firmas (De Silva, Rodrigues y Klann, 2017; Ferchichi 
Divulgación de información no financiera sobre capital intelectual en el reporte integrado...

y Paturel, 2013; Ficco et al., 2021a; Ficco y Sader, 2020; Kimouche y Rouabhi, 2016; Nimtrakoom, 2015; Ocak y Findik, 2019; Oliveira, Rodrigues y Craig, 2010).

En la línea de los trabajos que se han centrado en estudiar la relevancia valorativa del $\mathrm{Cl}$ en el sector financiero, las evidencias son aún más contundentes. En este sentido, se destacan los resultados que revelan que todas las dimensiones del $\mathrm{Cl}$ tienen relevancia para la valoración de los bancos (Irsyahma y Nikmah, 2017; Ozkan, Cakan y Kayakan, 2017; Salvi et al., 2020; Veltri y Silvestri, 2011), lo que confirma la importancia que posee el $\mathrm{CI}$ en este sector, que es uno de los más intensivos en conocimiento y en el que el $\mathrm{Cl}$ generalmente representa un recurso crítico para la creación de valor (Alonso, Saraite, Haro de Rosario y Caba-Pérez, 2016; Saengchan, 2008).

Sin embargo, aun cuando la evidencia empírica revela claramente el rol del CI en los procesos de generación de valor de las empresas y el RI es, precisamente, un informe destinado a explicar cómo se produce la creación de valor a lo largo del tiempo, los estudios que han considerado a este informe como fuente de divulgación de $\mathrm{Cl}$ son comparativamente más escasos y, además, refieren, principalmente, a empresas que operan en el contexto de mercados de capitales desarrollados.

En esta línea, Melloni (2015) analizó el contenido y los atributos linguiísticos, como indicadores de calidad, de la información sobre $\mathrm{Cl}$ que se divulga en el RI, a través de la técnica de análisis de contenido. Los resultados obtenidos muestran que las empresas comunican información referida mayoritariamente a los capitales Social y Relacional y Financiero del MIRI. En relación con la divulgación sobre $\mathrm{CI}$, concluye que la información en los RI se enfoca principalmente en la dimensión de $\mathrm{CR}$, seguida por la de $\mathrm{CH}$, siendo el $\mathrm{CE}$ el menos reportado.

Utilizando una estrategia metodológica similar, Beretta, Demartini y Trucco (2019) estudiaron la asociación entre el tono de la información sobre Cl en el RI y el desempeño financiero y no financiero. En este caso, las evidencias obtenidas revelan marcadas diferencias entre las dimensiones del $\mathrm{Cl}$ divulgadas en los $\mathrm{RI}$, destacándose el $\mathrm{CH}$ y el CE como las categorías más y menos comunicadas, respectivamente.

Salvi, Vitolla, Giakoumelou, Raimo y Rubino (2020) realizan un estudio pionero en lo que respecta al examen del impacto de la divulgación de información sobre $\mathrm{Cl}$, realizada a través del RI, en el valor de las empresas. Sus hallazgos muestran, por un lado, que la calidad de la divulgación del CI dentro de los RI sigue siendo baja, aunque obtienen evidencia de una relación positiva entre el valor de la empresa y los tres componentes del $\mathrm{Cl}$ (estructural, humano y social y de relación) que se divulgan a través del RI.

En el caso particular del sector financiero, el estudio de Santis et al. (2019), analiza de qué manera las empresas de este sector divulgan información sobre los distintos componentes del $\mathrm{CI}$ en sus RI y cómo la vinculan con la creación de 
valor. Los principales hallazgos revelan, por un lado, que las empresas brindan información sobre $\mathrm{Cl}$ en el RI, adoptando una clasificación cercana a la que se emplea en la literatura específica de $\mathrm{CI}$. Por otro lado, detectan que la gran mayoría de las empresas investigadas tiende a adoptar un enfoque superficial, revelando escasa información sobre el vínculo entre el $\mathrm{Cl}$ y el proceso de creación de valor.

Birindelli, Ferretti, Chiappini y Cosentino (2020) analizan múltiples fuentes de información de CI publicadas por los bancos, entre las que se incluye el RI. Las principales evidencias obtenidas muestran que la información sobre $\mathrm{Cl}$ es limitada, tanto en contenido como en calidad, siendo la dimensión de CR la más revelada y, dentro de ella, la información referida a las relaciones con clientes.

De este modo, la revisión realizada muestra una profusa literatura empírica que ha analizado las prácticas voluntarias de divulgación del $\mathrm{Cl}$, como así también, la influencia del $\mathrm{Cl}$ en la creación de valor. Sin embargo, también refleja que los estados financieros son la fuente de información más utilizada para realizar esos análisis, siendo incipiente la literatura que estudia la divulgación del $\mathrm{CI}$ en el contexto del RI. Esta situación deja en claro la necesidad de avanzar en una temática cuyo abordaje se encuentra aún inconcluso, así como de brindar evidencia para el contexto latinoamericano. Con este fin, se desarrolla la investigación empírica que presentamos seguidamente.

\section{Estrategia metodológica}

El estudio empírico está basado en el enfoque del estudio de caso (Ryan, Scapens y Theobald, 2004; Yin, 2009). La adopción de este enfoque se sustenta en la postura de distintos autores que consideran que los estudios de caso son más apropiados para lograr una mejor comprensión acerca de cómo las organizaciones adaptan los RI a distintas necesidades (De Villiers, Rinaldi y Unerman, 2014; Doni, Larsen, Bianchi y Corvino, 2019).

En particular, se trata de un estudio de caso de tipo descriptivo, en tanto se pretende proporcionar una descripción de la práctica estudiada, brindando "información sobre la naturaleza y forma [de esa práctica)" (Ryan, Scapens y Theobald, 2004, p. 193), la que, en esta investigación, refiere a la divulgación de información no financiera sobre $\mathrm{CI}$ a través del RI.

En el apartado introductorio se mencionó que, en Argentina, solo tres empresas cotizantes, pertenecientes al sector financiero, cumplen actualmente la condición de publicar su RI elaborado a partir del marco de referencia del IIRC y, en particular, que una de ellas lo hace desde el año 2014; mientras que las restantes publican sus RI desde 2018. En este contexto, el BMA fue seleccionado para el estudio de caso planteado, por ser la empresa pionera en la presentación de RI elaborados en base al marco del IIRC en Argentina, manteniendo tal política de divulgación de información desde 2014, de manera ininterrumpida. 
Divulgación de información no financiera sobre capital intelectual en el reporte integrado...

En lo que respecta a la dimensión temporal, se trabajó con el período 20142019. El período de análisis inicia en 2014, por ser este ejercicio el que corresponde a la primera publicación del RI del BMA, y finaliza en 2019, que es el último ejercicio cerrado al momento de realizar esta investigación.

Los RI publicados por el BMA fueron estudiados a partir de la metodología de análisis de datos textuales (textual analysis), en tanto permite investigar cómo un fenómeno o práctica empresarial es comunicado a través de un medio escrito (Benites et al., 2017). Se trata así de una técnica apropiada para analizar las prácticas de revelación voluntaria de información no financiera sobre $\mathrm{CI}$ en un documento escrito, como es el RI.

El análisis de datos textuales comprende un conjunto de herramientas estadísticas aplicadas al estudio multidimensional de datos linguíísticos (Franzosi, 2010), las cuales se integran con técnicas cualitativas de análisis interpretativo para describir el contenido de un conjunto de textos referidos a un objeto de estudio específico (Vaara, 2010). Este enfoque metodológico considera, en el análisis del corpus $^{1}$, tanto la frecuencia de uso de las palabras o formas en el texto, como las conexiones entre las mismas dentro del contexto en que son utilizadas. De este modo, se diferencia del tradicional análisis de contenido, que es la metodología de uso habitual en los estudios sobre divulgación de información de Cl.

El análisis textual sobre los RI del BMA fue realizado utilizando el software IRaMuTeQ (Interfaz de R para el Análisis Multidimensional de los Textos y Cuestionarios). La utilización de este software permite el procesamiento de gran cantidad de datos no estructurados de manera fiable, reduciendo la subjetividad implícita en la codificación manual de textos. En este sentido, el análisis de textos a través de IRaMuTeQ asegura que los resultados obtenidos permanecen constantes, independientemente de las circunstancias en que se lleve a cabo; de este modo, se garantizan las condiciones de estabilidad y reproducibilidad del método de análisis en los términos de Krippendorff (2018).

En particular, se aplicó el método de análisis de similitud, el cual examina la estructura temática subyacente del corpus textual a partir de la identificación de núcleos temáticos detectados por las coocurrencias ${ }^{2}$ de las palabras y sus conexiones en el texto (Marchand y Ratinaud, 2012). La identificación de estos núcleos o clases temáticas se logra a través de un profundo análisis cuantitativo del texto que se realiza con el apoyo del software referido, el cual aporta una representación gráfica del contenido y organización interna del texto analizado, poniendo de manifiesto las clases temáticas y los vínculos entre los elementos que componen la representación del objeto de estudio. Sin embargo, como

1 El término "corpus" se refiere al conjunto de textos referidos al objeto de estudio de una investigación.

2 En lingüística, se entiende por "coocurrencia" a la utilización conjunta de palabras dentro de un corpus. Constituye un indicador de la interdependencia o proximidad semántica entre palabras. 
advierte Vaara (2010), estos resultados deben interpretarse desde el punto de vista cualitativo; en otras palabras, debe asignarse "significado" a los resultados sobre la base del conocimiento del investigador sobre la temática y teniendo en cuenta el marco teórico de la investigación y los aportes de la literatura previa.

El corpus para este estudio se conforma con los RI publicados por el BMA en el período 2014 a 2019, los cuales fueron recuperados de la página web corporativa del banco ${ }^{3}$. El análisis se enfoca en la sección de los RI denominada "reporte anual" o "memoria anual" y, específicamente, en aquellos apartados referidos a cada uno de los capitales del MIRI, los cuales fueron analizados como subcorpus.

\section{Resultados y discusión}

\section{IV.1 Presentación del caso: Banco Macro S.A.}

El BMA es una empresa líder en el sector financiero de Argentina, siendo el banco privado de mayor capitalización bursátil en el mercado de capitales local $^{4}$. Este banco fue pionero en la presentación de RI como estrategia de comunicación de sus políticas, acciones y su desempeño económico, social y ambiental. La adopción del RI le permitió al BMA integrar, en un único informe, información financiera y no financiera referida a "la construcción de valor desde una dimensión transversal de los recursos utilizados"; destinada a un amplio espectro de stakeholders, a saber: "clientes, proveedores, colaboradores, organizaciones aliadas, sector público, inversionistas, analistas financieros y medios de comunicación” (BMA, 2014, p. 7).

\section{IV.2 La información no financiera sobre el CI y sus distintas dimensiones en los RI del BMA}

En esta sección se presentan los resultados del análisis de similitud, el cual permitió identificar las temáticas subyacentes en los subcorpus referidos a cada uno de los capitales del MIRI, a partir del análisis de las palabras significativamente asociadas a cada apartado y con mayor frecuencia de ocurrencia. Por otro lado, se determinaron las conexiones entre esas clases temáticas en los textos, teniendo en cuenta la coocurrencia de las palabras que las caracterizan. Estos resultados se presentan a través de los gráficos de similitud (Figuras 2 a 8), en los que los núcleos o nodos indican conjuntos temáticos, mientras que los enlaces entre ellos representan las conexiones entre los mismos. Además, siguiendo a Vaara (2010), se trabajó en la interpretación de los gráficos de similitud, apelando al marco teórico de la investigación y a los aportes de la literatura previa. Esto permitió determinar el sentido de las palabras y sus

3 https://www.macro.com.ar/

4 Fuente: www.bolsar.info. La capitalización bursátil de las empresas listadas en el mercado de capitales argentino fue obtenida a la fecha de realización de este estudio (febrero de 2021). 
Divulgación de información no financiera sobre capital intelectual en el reporte integrado...

relaciones en su contexto de uso, para poder establecer conexiones entre la información divulgada en los RI y las tres dimensiones que define la literatura clásica para el $\mathrm{Cl}$.

La Figura 2 expone el gráfico de similitud para "Capital Financiero", el cual comprende los "recursos económicos y financieros con los que cuenta Banco Macro para el desempeño de sus acciones" (BMA, 2016, p. 67). Este capital ha sido tradicionalmente el principal foco de divulgación en el sector bancario (IIRC, 2015). En el caso del BMA, la información sobre Capital Financiero se estructura alrededor de cuatro núcleos temáticos. El núcleo central, cuya palabra clave es "privado", se relaciona estrechamente con el nodo caracterizado por el término "préstamo". Así, se advierte que estos núcleos se refieren a recursos críticos para el desarrollo de la actividad del BMA: los depósitos del sector privado y su cartera de préstamos. Estos nodos se vinculan, por un lado, a un núcleo conformado por la coocurrencia de términos referidos a su desempeño económico-financiero, siendo las más significativas "financiero", "resultado" y "mercado". Por otro lado, los recursos de BMA se asocian al nodo que evoca la situación patrimonial del "banco" y su vínculo con la autoridad de contralor ("BCRA").

Figura 2. Gráfico de similitud para Capital Financiero - BMA.

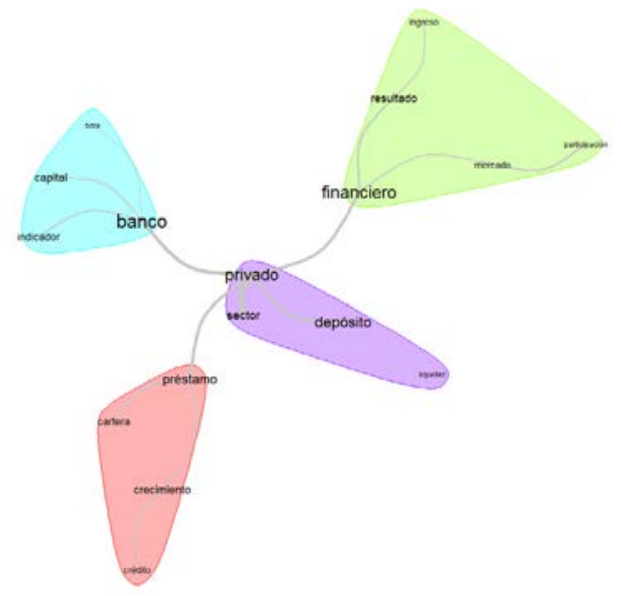

Fuente: elaboración propia.
Figura 3. Gráfico de similitud para Capital Industrial - BMA.

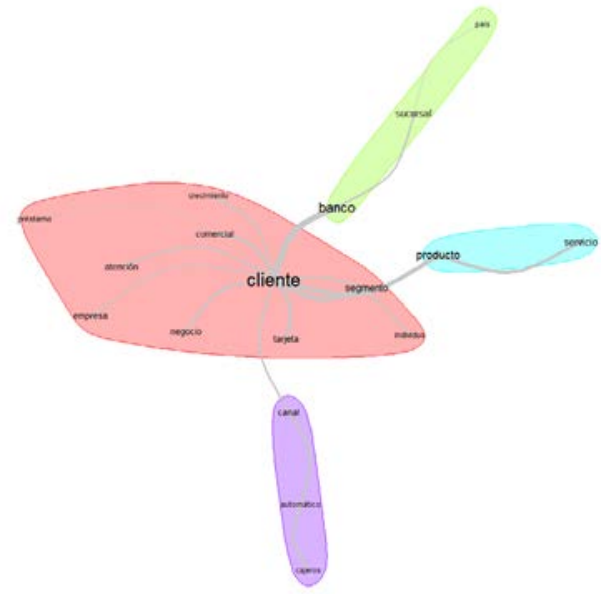

Fuente: elaboración propia.

La Figura 3 presenta el gráfico de similitud para "Capital Industrial”, en el cual se destaca la centralidad de un núcleo temático asociado al término "cliente", referido a los distintos segmentos de usuarios del banco. Este núcleo se enlaza con tres nodos vinculados con aspectos mencionados por el BMA en

5 BCRA: Banco Central de la República Argentina. 
sus RI como integrantes de su Capital Industrial: productos y servicios, activos tangibles, evocados en el gráfico a través de las palabras "canal", "automático" y "cajeros"; y presencia geográfica en Argentina, relacionado a los términos "banco", "sucursales", y "país". De este modo se advierte que la información sobre Capital Industrial es reportada por el BMA desde una perspectiva orientada al cliente, evidenciando su importancia.

La Figura 4 muestra el gráfico de similitud para "Capital Intelectual", el cual comprende "los activos intangibles relacionados con nuestra reputación, el desarrollo y know-how de la organización" (BMA, 2016, p.97). En este apartado se identifican cuatro núcleos temáticos. Por un lado, se observan dos nodos ubicados a los extremos del gráfico: un núcleo relacionado a la gestión de la "experiencia" del cliente del BMA, y otro vinculado a los canales de "atención" del banco ("sucursal", "telefónico", "gestión" de "reclamos", "autogestión"). Estos núcleos se vinculan a través de dos nodos centrales relacionados a los sistemas de información y de comunicación ("digital”, "internet", "app”). Asimismo, se destaca la coocurrencia entre los términos "digital" y "transformación”, lo cual puede relacionarse al fenómeno de creciente digitalización y desintermediación que caracteriza al sector bancario (Birindelli et al., 2020; IIRC, 2015; Mention, 2011), y que ha incrementado la relevancia de capitales no financieros, como el intelectual, para crear ventajas competitivas (Doni et al.,2019, IIRC,2015).

De este modo, se advierte, en línea con Santis et al. (2019), que la información sobre Capital Intelectual del BMA se relaciona con la dimensión estructural del $\mathrm{CI}$, en tanto involucra elementos intangibles asociados con los sistemas de información y comunicación y con la capacidad del BMA de "transformarse", adoptando nuevas tecnologías que le permitan ofrecer a sus clientes mejores experiencias y nuevas formas de atención. Si bien el CE tiende a ser la dimensión del CI menos divulgada por los bancos (Beretta et al., 2019; Melloni, 2015; Santis et al., 2019), Birindelli et al. (2020) observaron un incremento en la información sobre CE vinculado a la categoría "sistemas de comunicación", evidenciando su creciente centralidad en la relación entre los bancos y sus stakeholders, lo cual resultaría consistente con el caso del BMA.

En la Figura 5 se expone el gráfico de similitud para "Capital Humano", el cual se estructura alrededor de un núcleo temático central, caracterizado por la palabra "colaborador". En los RI analizados, el personal del BMA es designado como "colaborador", terminología utilizada también por otros bancos argentinos (Ficco et al., 2021b). Este núcleo central se vincula con otros seis nodos, referidos a distintos aspectos de la relación entre el BMA y sus colaboradores. Por un lado, se observa un núcleo caracterizado por las palabras "hombre" y "mujer", evocando cuestiones de "género", lo cual puede considerarse como una adaptación del RI a las demandas informativas de nuestros tiempos. Asimismo, "colaborador" coocurre con "desarrollo", "profesional" y "gestión", términos 
característicos de tres núcleos temáticos asociados con aspectos referidos a formación y desarrollo profesional del personal del BMA y con la medición de su desempeño. Por otro lado, se observan, dos nodos relacionados con el bienestar de los colaboradores, en términos de "salud" y "seguridad" "laboral" y de participación en "programas", como el caso de "voluntariados", respectivamente.

Así, el Capital Humano del BMA se vincula estrechamente con la dimensión análoga del $\mathrm{Cl}$, en tanto refiere al valor intangible que reside en los empleados de la firma (Santis et al., 2019). El CH desempeña un rol crítico en el sector bancario, en tanto los bancos dependen de los conocimientos y habilidades de sus "colaboradores" (IIRC, 2015; Mention, 2011), motivo por el cual es considerado como el "factor de crecimiento" del banco (BMA, 2016, p.107). Además, se identifican en este apartado elementos mencionados en la literatura como integrantes del $\mathrm{CH}$, tales como: actividades de formación y desarrollo de competencias, salud y seguridad, y diversidad e inclusión laboral.

Figura 4. Gráfico de similitud para Capital Intelectual - BMA.

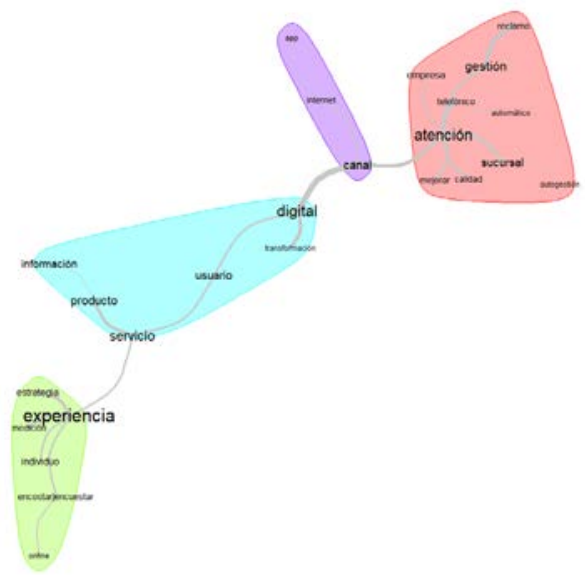

Fuente: elaboración propia.
Figura 5. Gráfico de similitud para Capital Humano - BMA.

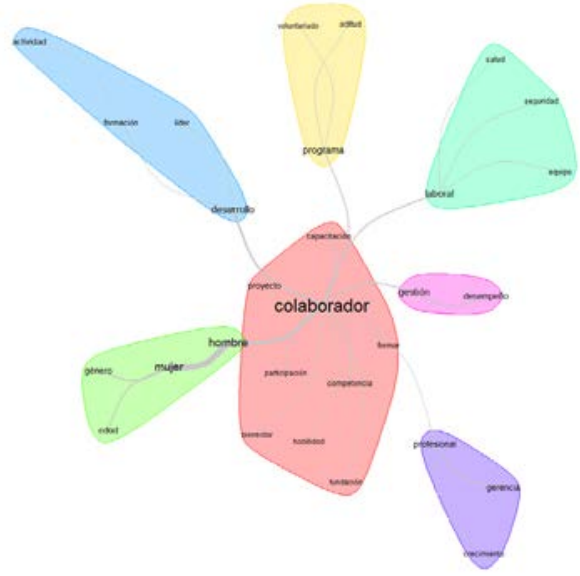

Fuente: elaboración propia.

En la Figura 6 se presenta el gráfico de similitud para "Capital Social y Relacional", conformado por cuatro núcleos temáticos vinculados entre sí a través de la palabra "programa". Este término evoca a las acciones que lleva adelante el banco para construir su "reputación e imagen corporativa" (BMA, 2016, p. 117). En este sentido, cada núcleo se refiere a las distintas iniciativas desarrolladas por el BMA en su ámbito territorial ("provincias" y "localidades"). Así, por un lado, se observa que el "banco" se relaciona con "proveedores" "locales", propendiendo al "desarrollo" de la "comunidad". Por otro lado, lleva adelante programas de "educación" e "inclusión" "financiera", y programas "sociales". Este tipo de esfuerzos, tendientes a construir la reputación corporativa, han sido señalados por Doni et al. (2019) 
y el IIRC (2015) como una consecuencia de la pérdida de confianza en los bancos ocasionada por las crisis financieras.

De este modo, el Capital Social y Relacional se refiere a los elementos intangibles que pueden generar valor a partir de las relaciones del BMA con sus stakeholders, tales como proveedores, clientes y la comunidad en general. En tal sentido, este capital se vincula a la dimensión relacional del $\mathrm{Cl}$; asociación en línea con los hallazgos de Santis et al. (2019).

Por último, la Figura 7 ilustra el gráfico de similitud para "Capital Natural", el cual se estructura en tres núcleos temáticos. El nodo principal, en términos de la significatividad y frecuencia de sus palabras asociadas, se relaciona con la "gestión" del "impacto ambiental directo e indirecto" del banco (BMA, 2016, p. 131). La gestión ambiental del BMA se enfoca en dos aspectos, evocados por sendos núcleos temáticos: la reducción en la utilización de insumos, tales como "papel” y "plástico", por un lado, y la disminución en el "consumo" de "energía", por el otro.

A pesar de que los bancos no se caracterizan por el uso intensivo de recursos naturales, su inclusión es frecuente en sus RI (IIRC, 2015). En el caso del BMA, la información divulgada en este apartado se refiere a los procesos medioambientales y, en ese sentido, de acuerdo con Ficco et al. (2021b), puede relacionarse con la dimensión estructural del Cl.

Figura 6. Gráfico de similitud para Capital Social y Relacional - BMA.

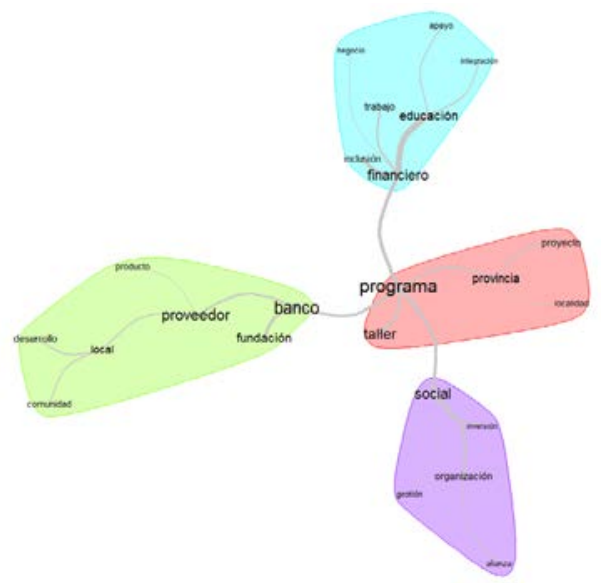

Fuente: elaboración propia.
Figura 7. Gráfico de similitud para Capital Natural - BMA.

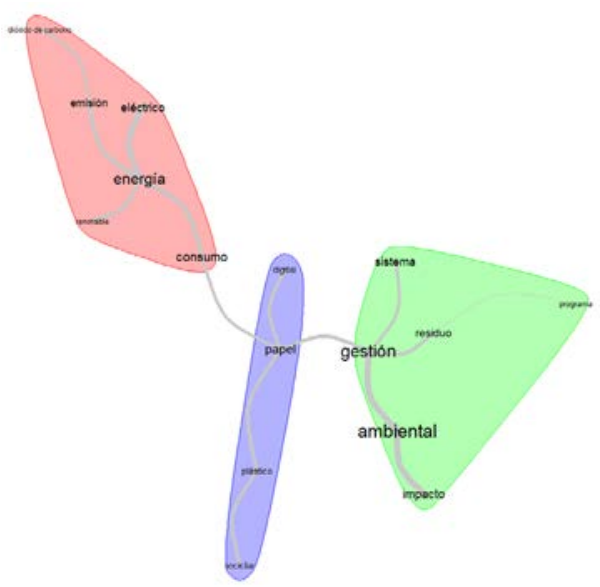

Fuente: elaboración propia.

Los resultados obtenidos a partir de la interpretación de los gráficos de similitud referidos a los capitales del MIRI evidencian que los RI del BMA incluyen información no financiera sobre $\mathrm{CI}$, lo que se encuentra en línea con los estudios previos (Beretta et al., 2019; Birindelli et al., 2020; Melloni, 2015; Salvi et al., 2020; Santis et al., 2019), apoyando la idea de que el CI está resurgiendo 
Divulgación de información no financiera sobre capital intelectual en el reporte integrado...

como parte del RI (De Villiers et al., 2014). Se aprecia asimismo que, aunque esta información se presenta bajo la estructura de capitales del MIRI, en el caso analizado se verifican empíricamente las asociaciones identificadas, a nivel conceptual, entre esos capitales y las tres dimensiones que se reconocen en la literatura específica del Cl. Así, el $\mathrm{CH}$ se vincula directamente al conocimiento intrínseco de los "colaboradores" del BMA, y a su desarrollo profesional y personal, como se destaca en el apartado sobre Capital Humano. Por su parte, el conocimiento explícito del banco y su capacidad para innovar y desarrollar nuevas propuestas de valor adaptándose a los cambios en el entorno, comunicadas en el apartado sobre Capital Intelectual, responden a la dimensión de CE. Asimismo, también pueden asociarse a esta dimensión las políticas medioambientales BMA, informadas en el apartado sobre Capital Natural. Por su parte, el CR se presenta como Capital Social y Relacional, en tanto refiere al valor de la reputación del banco y a las relaciones con sus múltiples stakeholders.

Asimismo, la ausencia de elementos relacionados al $\mathrm{Cl}$ en las secciones referidas a Capital Financiero y Capital Industrial, está en sintonía con los resultados de Ficco et al. (2021b) y Santis et al. (2019), en tanto tales capitales no tienen relación con las dimensiones clásicas del $\mathrm{CI}$, por estar principalmente vinculados a elementos tangibles necesarios para la creación de valor, en contraposición a la característica de intangible del $\mathrm{CI}$.

\section{Conclusiones}

Frente a las limitaciones de la contabilidad financiera, para el reporte de información referida al $\mathrm{Cl}$, la divulgación voluntaria de información no financiera, a través de diversos medios y canales, permite a las empresas comunicar a terceros la relevancia de estos recursos, actividades y su importante papel en la generación de valor. En este contexto, el RI propuesto por el IIRC se posiciona como un instrumento que permite a las organizaciones comunicar cómo crean valor a lo largo del tiempo, a partir de la integración de recursos o capitales de distinta naturaleza, dentro de los cuales se encuentra el CI. Sin embargo, la divulgación de información sobre $\mathrm{Cl}$ en el RI es una temática escasamente estudiada, particularmente en el contexto de mercados emergentes, como es el caso de Latinoamérica.

En este marco, el presente artículo tuvo como objetivo fundamental analizar cómo se realiza la divulgación de información no financiera sobre $\mathrm{Cl}$ en los RI que se elaboran en base al marco de referencia del IIRC. Para dar cumplimiento al mismo, se realizó un estudio empírico basado en el enfoque del estudio de caso. El caso analizado corresponde al BMA, un banco pionero en la presentación de RI, elaborados a partir del MIRI, en el mercado de capitales argentino. El estudio se realizó sobre los RI publicados por el BMA correspondientes al período 2014-2019, aplicando la metodología de análisis de datos textuales y, en 
particular, el método de análisis de similitud. Esta metodología, novedosa en el campo de la contabilidad, permitió analizar la estructura y el contenido de la información sobre capitales, reportada en los RI, integrando técnicas estadísticas con la interpretación del significado de estos textos en su contexto de uso.

Los hallazgos obtenidos indican que el BMA divulga información no financiera sobre $\mathrm{CI}$, y sus dimensiones, humana, estructural y relacional, en el RI, adoptando la estructura del marco del IIRC. En concreto, el $\mathrm{CH}$ se vincula estrechamente con su versión análoga en el MIRI, identificándose la utilización de un término particular ("colaborador") para designar al componente humano del banco y la presencia de temas vinculados a cuestiones de género, a formación y desarrollo profesional del personal y a su bienestar. El CE se hizo presente tanto en la sección referida a Capital Intelectual, vinculado a los elementos intangibles asociados con los sistemas de información y comunicación y con la capacidad de innovar, como en la de Capital Natural, en relación con las políticas medioambientales del BMA. El CR fue reportado en la sección de Capital Social y Relacional, destacando la información referida a las iniciativas desarrolladas por el BMA en su ámbito territorial, encaminadas a la construcción de su reputación corporativa.

Los resultados obtenidos aportan evidencia al campo de la investigación sobre divulgación voluntaria de información de $\mathrm{Cl}$, y, en particular, de la que se canaliza a través del RI, cuyo desarrollo es aún incipiente. Esta evidencia es reveladora del modo en que las prácticas de divulgación sobre $\mathrm{CI}$ se pueden adaptar al enfoque del RI. Asimismo, la pertenencia del caso analizado al sector bancario, permite complementar los hallazgos de aquellos trabajos que analizan las prácticas de divulgación de este sector, el cual es frecuentemente excluido en la literatura. Además, dado que el estudio se contextualiza en el mercado de capitales argentino, el mismo es un aporte al conocimiento de las políticas de comunicación del $\mathrm{Cl}$ en el RI para el contexto latinoamericano, donde el RI es una práctica de escaso desarrollo. Finalmente, el trabajo también contribuye a enriquecer la línea de investigación en la que se enmarca en sus aspectos metodológicos, en la medida que propone una metodología novedosa: el análisis de datos textuales, el cual puede ser un importante complemento del tradicional análisis de contenido.

Respecto de las implicaciones prácticas de este estudio, la investigación contribuye al conocimiento de cómo una empresa pionera en la adopción del RI ha interpretado y adaptado los conceptos del MIRI a sus estrategias de comunicación sobre sus capitales, y en particular en relación con su $\mathrm{Cl}$, con la finalidad de satisfacer las distintas necesidades informativas de sus stakeholders. Asimismo, la evidencia empírica obtenida en el caso de estudio contrasta la asociación entre los capitales del MIRI y las dimensiones del CI señalada en la literatura previa. En ese sentido, el estudio también contribuye a acrecentar los aportes 
Divulgación de información no financiera sobre capital intelectual en el reporte integrado...

de la investigación en torno al $\mathrm{Cl}$, los que podrían ser considerados por el IIRC para revisar los conceptos y alcances de los capitales del MIRI, de manera tal de posicionar al RI como un instrumento apropiado para la divulgación sobre $\mathrm{CI}$ y su capacidad de generar valor en relación con otros capitales.

No obstante, a la hora de analizar estas conclusiones, es necesario tener en cuenta que la investigación tiene ciertas limitaciones. Por un lado, al tratarse de un estudio de caso, las evidencias de este estudio deben analizarse en el contexto especifico de su realización, no pudiendo generalizarse. Por otro lado, en el mercado de capitales argentino solo un reducido número de empresas del sector bancario publica sus RI, lo que constituye una limitación para contrastar los hallazgos de este estudio con otros sectores de actividad.

Por ello, de cara a una futura investigación, resultaría valioso analizar las prácticas de divulgación del $\mathrm{Cl}$ en otros países, donde el RI sea una práctica empresarial más generalizada. Asimismo, dadas las limitaciones en términos de la cantidad de empresas que publican RI en Argentina, otra propuesta de investigación futura consiste en profundizar el estudio empírico analizando otros medios corporativos de divulgación voluntaria de información sobre $\mathrm{Cl}$, como los reportes anuales y las memorias de sostenibilidad. Finalmente, resultaría interesante complementar los resultados obtenidos a través de la metodología de análisis de datos textuales, con la técnica de análisis de contenido, con la finalidad de construir indicadores relativos al contenido y atributos de la información divulgada sobre $\mathrm{CI}$, en línea con otros estudios previos en la temática. De este modo, se profundizaría el análisis de la naturaleza y extensión de la divulgación sobre $\mathrm{Cl}$ en el RI, como paso previo para indagar respecto de relevancia del $\mathrm{Cl}$ en creación de valor empresarial, concepto central en el RI.

\section{Referencias bibliográficas}

Abhayawansa, S., Guthrie, J. y Keynes, M. (2019). Intellectual capital accounting in the age of integrated reporting: a commentary. Journal of Intellectual Capital, 20(1), 2-10. DOI: 10.1108/JIC-01-2019-223

Al-Hajaya, K., Altarawneh, M. y Altarawneh, B. (2019). Intellectual Capital Disclosure by Listed Companies in Jordan: A Comparative Intersector Analysis. International Review of Management and Marketing, 9(1), 109-116. DOI: 10.32479/irmm.7381

Alonso, J., Saraite, L., Haro de Rosario, A. y Caba-Pérez, C. (2016). Sector bancario a nivel mundial y los factores que influyen en su información sobre responsabilidad social corporativa. Contaduría Universidad de Antioquia, (68), 213-233.

Badia, F., Dicuonzo, G., Petruzzelli, S. y Dell'Atti, V. (2019). Integrated reporting in action: Mobilizing intellectual capital to improve management and governance practices. Journal of Management and Governance, 23(2), 299-320. DOI: 10.1007/s10997-018-9420-1

Banco Macro S.A. (2014). Reporte anual integrado Banco Macro S.A. 2014. Recuperado de: https://www.macro.com.ar/relaciones-inversores/informacion-financiera/informes-yreporte 
Banco Macro S.A. (2016). Reporte anual integrado Banco Macro S.A. 2016. Recuperado de: https://www.macro.com.ar/relaciones-inversores/informacion-financiera/informes-yreporte

Benites, L., Mello, N. y Lahsen, M. (2017). Business storytelling about energy and climate change: The case of Brazil's ethanol industry. Energy Research y Social Science, 31, 77-85. DOI: 10.1016/j.erss.2017.06.008.

Beretta, V., Demartini, C. y Trucco, S. (2019). Does environmental, social and governance performance influence intellectual capital disclosure tone in integrated reporting? Journal of Intellectual Capital, 20(1), 100-124. DOI: 10.1108/JC-02-2018-0049

Birindelli, G., Ferretti, P., Chiappini, H. y Cosentino, A. (2020). Intellectual Capital Disclosure: Some Evidence from Healthy and Distressed Banks in Italy. Sustainability, 12(8), 3174. DOI: $10.3390 /$ su 12083174

Blaise, S., Carson, K. y Phillips, P. (2008). Intellectual capital disclosure by traditional US companies: A longitudinal assessment. Journal of Accounting and Organizational Change, 4(1), 67-80. DOI: 10.1108/18325910810855798

Bollen, L., Vergauwen, P. y Schnieders, S. (2005). Linking intellectual capital and intellectual property to company performance. Management Decision, 43(9), 1161-1185. DOI: $10.1108 / 00251740510626254$

Bontis, N. (1998). Intellectual capital: An exploratory study that develops measures and models. Management Decision, 36(2), 63-76. DOI: 10.1108/00251749810204142

Bueno, E., Salmador, M. y Merino, C. (2008). Génesis, concepto y desarrollo del capital intelectual en la economía del conocimiento: Una reflexión sobre el Modelo Intellectus y sus aplicaciones. Estudios de Economía Aplicada, 26(2), 43-63.

Busco, C., Malafronte, I. Pereira, J. y Starita, M. (2019). The determinants of companies' levels of integration: Does one size ft all? British Accounting Review, 51(3), 277-298. DOI: 10.1016/j.bar.2019.01.002

Cañibano, L. (2012). La relevancia de los intangibles en la información financiera. Contaduría Universidad de Antioquia, (60), 41-54.

Cañibano, L., Sánchez, M. García, M. y Chaminade, C. (2002). Directrices para la gestión y difusión de información sobre intangibles (Informe de Capital Intelectual). Proyecto Meritum. Madrid: Fundación Airtel Móvil.

Castilla, F. y Ruiz, C. (2018). La divulgación de intangibles en entidades financieras: un análisis de contenido. Revista Española de Financiación y Contabilidad, 47(1), 81-123. DOI: $10.1080 / 02102412.2017 .1346912$

Da Silva, A., Rodrigues, T. y Klann, R. (2017). A influência dos ativos intangíveis na relevância da informação contábil. Revista Contemporânea de Contabilidade, 14(31), 26-45. DOI: $10.5007 / 2175-8069.2017 \mathrm{v} 14 \mathrm{n} 31 \mathrm{p} 26$

De Silva, T., Stratford, M. y Clark, M. (2014). Intellectual capital reporting: a longitudinal study of New Zealand companies. Journal of Intellectual Capital, 15(1), 157-172. DOI: 10.1108/JIC-03-2013-0034

De Villiers, C., Rinaldi, L. y Unerman, J. (2014). Integrated Reporting: Insights, gaps and an agenda for future research. Accounting, Auditing y Accountability Journal, 27 (7), 1042-1067. DOI: 10.1108/AAAJ-06-2014-1736 
Divulgación de información no financiera sobre capital intelectual en el reporte integrado...

Dey, P. (2020). Value relevance of integrated reporting: a study of the Bangladesh banking sector. International Journal of Disclosure and Governance, 17, 195-207. DOI: 10.1057/s41310-020-00084-z

Doni, F., Larsen, M., Bianchi, S. y Corvino, A. (2019). Exploring integrated reporting in the banking industry: the multiple capitals approach. Journal of Intellectual Capital, 20(1), 165-188. DOI:10.1108/JIC-11-2017-0146

Dumay, J. (2016). A critical reflection on the future of intellectual capital: from reporting to disclosure. Journal of Intellectual Capital, 17(1), 168-184. DOI: 10.1108/JIC-08-20150072

Dumay, J. y Cai, L. (2014). A review and critique of content analysis as a methodology for inquiring into IC disclosure. Journal of Intellectual Capital, 15(2), 264-290. DOI: 10.1108/JIC-01-2014-0010

Edvinsson, L. y Malone, M. (1997). Intellectual Capital. Realizing your company's true value by findings its hidden brainpower. New York: Harper Collins Publishers.

Ferchichi, J. y Paturel, R. (2013). The effect of intellectual capital disclosure on the value creation: An empirical study using Tunisian annual reports. International Journal of Accounting and Financial Reporting, 3(1), 81-107. DOI: 10.5296/ijafr. v3i1.3238

Ficco, C. (2019). Los activos intangibles en la normativa contable argentina y en las normas internacionales de información financiera. Contabilidad y Auditoría, 50, 61-108.

Ficco, C. (2020). Una revisión del concepto de capital intelectual y de las principales alternativas para su identificación y medición. Revista Activos, 18(1), 1-42. DOI: $10.15332 / 25005278 / 6162$

Ficco, C., García, G., Sader, G. y Luna, J. (2021b). Capital intelectual en el reporte integrado: un estudio exploratorio en el mercado de capitales argentino. Revista Academia y Negocios, 7(2), p. 105-122.

Ficco, C. y Sader, G. (2020). Relevancia valorativa de información financiera y no financiera sobre capital intelectual en empresas cotizadas argentinas. CAPIC REVIEW: Journal of Accounting, Auditing and Business Management, 18, 1-16. DOI: 10.35928/cr. vol18.2020.87.

Ficco, C., Werbin, E., Díaz, M y Prieto, B. (2021a). Relevancia de los intangibles para la valoración de las acciones de las empresas en el mercado: evidencias desde el contexto argentino. Contaduría y Administración, 66(3), 1-26.

Franzosi, R. (2010). Quantitative Narrative Analysis. London: SAGE Publications.

García-Meca, E. (2005). Bridging the gap between disclosure and use of intellectual capital information. Journal of Intellectual Capital, 6(3), 427-440. DOI: 10.1108/14691930510611157

Ginesti, G., Caldarelli, A. y Zampella, A. (2018). Exploring the impact of intellectual capital on company reputation and performance. Journal of Intellectual Capital, 19(5), 915934. DOI: 10.1108/JIC-01-2018-0012

Herrera, E. y Macagnan, C. (2016). Revelación de informaciones sobre capital estructural organizativo de los bancos en Brasil y España. Contaduría y Administración, 61(1), 4-25. DOI: 10.1016/j.cya.2015.09.007

IIRC (2013). The international IR framework. London: IIRC. 
IIRC (2015). Applying the Integrated reporting concept of 'capitals' in the banking industry. $<$ IR > Banking Network. Recuperado de: http://integratedreporting.org

Irsyahma, A. y Nikmah, N. (2017). Intellectual capital, firm value and financial performance. AFEBI Accounting Review, 1(01), 29-43.

Kimouche, B. y Rouabhi, A. (2016). The impact of intangibles on the value relevance of accounting information: Evidence from French companies. Intangible Capital, 12(2), 506-529. DOI: 10.3926/ic.653

Krippendorff, K. (2018). Content analysis: An introduction to its methodology. Thousand Oaks, CA: Sage Publications.

Lentjušenkova, O. y Lapina, I. (2016). The transformation of the organization's intellectual capital: from resource to capital. Journal of Intellectual Capital, 17(4), 610-631. DOI: 10.1108/JIC-03-2016-0031

Macías, H. y Farfán, A. (2017). Integrated reporting as a strategy for firm growth: multiple case study in Colombia. Meditari Accountancy Research, 25(4), 605-628. DOI: 10.1108/ MEDAR-11-2016-0099

Marchand, P., y Ratinaud, P. (2012). L'analyse de similitude appliquée aux corpus textuels: les primaires socialistes pour l'élection présidentielle française (septembre-octobre 2011). En Actes des 11eme Journées internationales d'Analyse statistique des Données Textuelles. (pp. 687-699).

Martín, G., Navas, J., López, P., y Delgado, M. (2010). El capital intelectual de la empresa. Evolución y desarrollo futuro. Economía Industrial, 378, 37-44.

Melloni, G. (2015). Intellectual capital disclosure in integrated reporting: an impression management analysis. Journal of Intellectual Capital, 16(3), 661-680. DOI: 10.1108/ JIC-11-2014-0121

Mention, A. (2011). Exploring voluntary reporting of intellectual capital in the banking sector. Journal of Management Control, 22, 279-309. DOI: 10.1007/s00187-011-0139-2

Nimtrakoon, S. (2015). The relationship between intellectual capital, firms' market value and financial performance: Empirical evidence from the ASEAN. Journal of Intellectual Capital, 16(3), 587-618. DOI: 10.1108/JIC-09-2014-0104

Nonaka, I. (1991). The knowledge creating company. Harvard Business Review, 69, 96-104.

Ocak, M. y Findik, D. (2019). The impact of intangible assets and sub-components of intangible assets on sustainable growth and firm value: Evidence from Turkish listed firms. Sustainability, 11(19), 5359. DOI: 10.3390/su11195359

Ochoa, M., Prieto, B. y Santidrián, A. (2010). Estado actual de los modelos de capital intelectual y su impacto en la creación de valor en empresas de Castilla y León. Revista de Investigación Económica y Social de Castilla y León, 13, 15-205.

Oliveira, L., Rodrigues, L. y Craig, R. (2010). Intangible assets and value relevance: Evidence from the Portuguese stock exchange. The British Accounting Review, 42(4), 241-252. DOI: 10.1016/j.bar.2010.08.001

Ozkan, N., Cakan, S. y Kayacan, M. (2017). Intellectual capital and financial performance: A study of the Turkish Banking Sector. Borsa Istanbul Review,17(3), 190-198. DOI: 10.1016/j.bir.2016.03.001

Contaduría Universidad de Antioquia - No. 79. Medellín, julio-diciembre de 2021 
Divulgación de información no financiera sobre capital intelectual en el reporte integrado...

Pastor, D., Glova, J., Lipták, F. y Kovác, V. (2017). Intangibles and methods for their valuation in financial terms: Literature review. Intangible capital, 13(2), 387-410. DOI: 10.3926/ic.752

Rivera, Y., Zorio, A. y García, M. (2016). El concepto de informe integrado como innovación en reporting corporativo. Journal of Innovation \& Knowledge, 1(3). 144-155. DOI: 10.1016/j.jik.2016.01.016

Roos, G., Roos, J., Dragonetti, N. y Edvinsson, L. (2001). Capital Intelectual. Buenos Aires: Paidós.

Rossi, M., Festa, G., Chouaibi, S., Fait, M. y Papa, A. (2021). The effects of business ethics and corporate social responsibility on intellectual capital voluntary disclosure. Journal of Intellectual Capital, 22(7), 1-23. DOI: 10.1108/JIC-08-2020-0287

Ryan, B., Scapens, R. y Theobald, M. (2004). Metodología de la Investigación en Finanzas y Contabilidad. Barcelona: Deusto.

Saengchan, S. (2008). The role of intellectual capital in creating value in the banking industry. International Review of Business Research, 7(2), 157-169.

Salvi, A., Vitolla, F., Giakoumelou, A., Raimo, N. y Rubino, M. (2020). Intellectual capital disclosure in integrated reports: the effect on firm value. Technological Forecasting and Social Change, 160, 228-235. DOI: 10.1016/j.techfore.2020.120228.

Santis, S., Bianchi, M., Incollingo, A., y Bisogno, M. (2019). Disclosure of intellectual capital components in integrated reporting: An empirical analysis. Sustainability, 11(1), 6277. DOI: $10.3390 /$ su 11010062

Sardo, F. y Serrasqueiro, Z. (2018). Intellectual capital, growth opportunities, and financial performance in European firms: Dynamic panel data analysis. Journal of Intellectual Capital, 19(4), 747-767. DOI: 10.1108/JIC-07-2017-0099

Sharma, N. (2012). Intangible assets: A study of valuation methods. BVIMR Management Edge, 5(1), 61-69.

Sihotang, P. \& Sanjaya, Y. (2014). Reporting intellectual capital in annual reports: Evidence from Indonesia. Indonesian Capital Market Review, 1(2), 125-152. DOI: 10.21002/ icmr. v1i2.3653

Sveiby, K. (1997). The new organizational wealth. Managing and measuring knowledgebased assets. San Francisco: Berrett- Koehler Publishers.

Tejedo, F. (2016). La información de los recursos intangibles ocultos: ¿memorias de sostenibilidad o informe anual? European Research on Management and Business Economics, 22(2), 101-109. DOI: 10.1016/j.iedee.2015.06.001

Vaara, E. (2010). Taking the linguistic turn seriously: Strategy as A multifaceted and interdiscursive phenomenon. Advances in Strategic Management, 27, 29-50. DOI: 10.1108/S0742-3322(2010)0000027005

Veltri, S. y Silvestri, A. (2011). Direct and indirect effects of human capital on firm value: Evidence from Italian companies. Journal of Human Resource Costing y Accounting, 15(3), 232-254. DOI: 10.1108/14013381111178596

Yin, R. (2009). Case Study Research: Design and Methods. Thousand Oaks, California: Sage Publications.

Zambon, S. (2016). Ten years after: The past, the present and the future of scholarly investigation on intangibles and intellectual capital. Journal of Intellectual Capital, 17(1), 1-10. DOI: 10.1108/JIC-11-2015-0093 